\title{
MEASURABILITY AND CONTINUITY CONDITIONS FOR NONLINEAR EVOLUTIONARY PROCESSES
}

\author{
JOHN M. BALL
}

\begin{abstract}
This paper generalizes to nonlinear evolutionary processes on a metric space the well-known results connecting measurability and continuity properties with respect to time of linear semigroups of continuous operators on a Banach space.
\end{abstract}

1. Introduction. Let $X$ be a topological space. By definition, an evolutionary process on $X$ is a family of operators $U(t, s): X \rightarrow X$, defined for $t \in \mathbf{R}^{+}, s$ $\in \mathbf{R}$ and satisfying (i) $U(0, s)=$ identity; (ii) $U(t+\tau, s)=U(t, s+\tau) U(\tau, s)$ for $t, \tau \in \mathbf{R}^{+}, s \in \mathbf{R}$. Such processes arise in the mathematical modelling of nonautonomous systems, when $U(t, s) x$ represents the position (or state) at time $t+s$ of the point which at time $s$ was at $x$. In the special case when the operators $U(t, s) \stackrel{\text { def }}{=} T(t)$ are independent of $s$, the evolutionary process defines a semigroup $\{T(t)\}, t \in \mathbf{R}^{+}$. In the above $\mathbf{R}^{+}$denotes the nonnegative reals.

In [3] it was shown that in certain situations measurability and continuity properties known to be satisfied for a semigroup could be strengthened using the semigroup properties. We extend this work to evolutionary processes. Our Theorem 1 is, however, new even for semigroups for which it takes the following form.

THEOREM $1^{\prime}$. Let $\{T(t)\}, t \geqslant 0$, be a semigroup on a metric space $X$. If $T(t)$ is continuous for each $t \geqslant 0$, and if the map $t \mapsto T(t) x$ is strongly measurable on $(0, \infty)$ for each $x \in X$, then the map $(t, x) \mapsto T(t) x$ is continuous on $(0, \infty) \times X$.

Theorem 1' generalizes classical results due to von Neumann [17], Dunford [12] and Phillips [20] for the case when $X$ is a Banach space and each $T(t)$ is linear, and improves the result of Phillips (see Crandall and Pazy [8]) for $X$ Banach and $\{T(t)\}, t \geqslant 0$, a semigroup of nonexpansions. It is a Lebesgue measure counterpart for its category version due to Chernoff and Marsden [6], [7] (see also [3, Theorem 5.1]).

The other theorems of the paper follow in a straightforward way from those in [3]. Included are some counterexamples indicating directions in which the results cannot be improved.

2. Preliminaries. Throughout this section let $X$ be a metric space with metric $d$ and denote Lebesgue measure in $\mathbf{R}$ by $m$. A function $f:(0, \infty) \rightarrow X$ is said

Received by the editors December 10, 1974 and, in revised form, April 9, 1975.

AMS (MOS) subject classifications (1970). Primary 47A05; Secondary 34A10, 35B30.

Key words and phrases. Evolutionary processes, nonlinear semigroups, strong measurability, Lusin's theorem. 
to be strongly measurable if there exists a sequence $\left\{f_{n}\right\}$ of measurable countably-valued functions which converges almost everywhere to $f$ on $(0, \infty)$, and almost separably-valued if there exists a subset $E \subseteq(0, \infty)$ of zero measure such that $f((0, \infty) \backslash E)$ has a countable dense subset. It is easily shown (see Dunford and Schwartz [13, p. 147] for an analogous proof) that $f$ is strongly measurable if and only if (a) $f$ is almost separably-valued and (b) $f^{-1} U$ is Lebesgue measurable for every open $U \subseteq X$.

We need the following version of Lusin's theorem, our proof of which is adapted from that in Oxtoby [19].

Lemma 1. A function $f:(0, \infty) \rightarrow X$ is strongly measurable if and only if given any $\varepsilon>0$ there exists a closed set $F$ with $m((0, \infty) \backslash F)<\varepsilon$ such that $f$ is continuous when restricted to $F$.

Proof. Let $f$ be strongly measurable. Then there exists $E \subseteq(0, \infty)$ of zero measure such that $Z \stackrel{\text { eff }}{=} f((0, \infty) \backslash E)$ has a countable base of open sets $U_{i} \cap Z(i=1,2, \ldots)$ with $U_{i}$ open in $X$. For each $i$ there exists an open set $G_{i} \supseteq f^{-1} U_{i}$ such that $m\left(G_{i} \backslash f^{-1} U_{i}\right)<\varepsilon / 2^{i+1}$. Let $S=\cup_{i=1}^{\infty}\left(G_{i} \backslash f^{-1} U_{i}\right)$ so that $m(S)<\varepsilon / 2$. Let $U \subseteq X$ be open. Then $U \cap Z=\cup_{k}\left(U_{i_{k}} \cap Z\right)$ and

$$
f^{-1}(U \cap Z)=\bigcup_{k}\left(G_{i_{k}} \backslash S\right) \cap f^{-1} Z=\bigcup_{k} G_{i_{k}} \backslash(S \cup E)
$$

which is open in $(0, \infty) \backslash(S \cup E)$. There exists a closed set

$$
F \subseteq(0, \infty) \backslash(S \cup E)
$$

with $m((0, \infty) \backslash F)<\varepsilon$, and clearly $f$ restricted to $F$ is continuous.

Conversely let $F_{i}$ be closed sets with $m\left((0, \infty) \backslash F_{i}\right)<1 / i$ and such that $f$ is continuous when restricted to each $F_{i}$. Let $F=\cup_{i=1}^{\infty} F_{i}$. Then $m((0, \infty) \backslash F)$ $=0$ and $f(F)=\cup_{i=1}^{\infty} f\left(F_{i}\right)$ has a countable dense subset. Thus $f$ is almost separably-valued. Let $U \subseteq X$ be open. For each $i$ there exists an open set $G_{i} \subseteq(0, \infty)$ with $\left(f^{-1} U\right) \cap F_{i}=G_{i} \cap F_{i}$. Hence

$$
f^{-1} U=\left(\left(f^{-1} U\right) \backslash F\right) \cup \bigcup_{i=1}^{\infty}\left(G_{i} \cap F_{i}\right),
$$

which is clearly Lebesgue measurable.

3. Main results. Throughout this section we suppose that the evolutionary process $\{U(t, s)\}$ defined on the topological space $X$ satisfies the hypothesis:

(A) For each $t \in \mathbf{R}^{+}$the map $(s, x) \mapsto U(t, s) x$ is (jointly) sequentially continuous from $\mathbf{R} \times X \rightarrow X$.

Theorem 1. Let $X$ be a metric space. Suppose that for each $s \in \mathbf{R}, x \in X$ the map $t \mapsto U(t, s) x$ is strongly measurable on $(0, \infty)$. Then the map $(t, s, x)$ $\mapsto U(t, s) x$ is continuous on $(0, \infty) \times \cdot \mathbf{R} \times X$.

Proof. We first prove Theorem 1'. Let $x \in X$. We show that the map $f(t) \equiv T(t) x$ is continuous on $(0, \infty)$. The result then follows from a theorem of Chernoff and Marsden [6] (see also [3]). Let $0<a<a+\delta<\infty$ and denote by $I$ and $J$ the open intervals $(a, a+\delta)$ and $(a+\delta / 3, a+2 \delta / 3)$ respectively. Since $f$ is strongly measurable, by Lemma 1 there exists in $I$ a closed set $F_{r}$ of measure greater than $\delta-1 / r^{2}$ on which the restriction of $f$ is 
continuous. The continuity being uniform, there exists $\delta / 3>\eta_{r}>0$ such that $t, t+h \in F_{r}$ and $|h|<\eta_{r}$ imply that $d(f(t+h), f(t))<1 / r$. Fix $h_{r}$ with $\left|h_{r}\right|<\eta_{r}$. The set $\left\{t \in F_{r} \cap J: t+h_{r} \notin F_{r}\right\}$ has measure less than $1 / r^{2}$. Therefore $d\left(f\left(t+h_{r}\right), f(t)\right)<1 / r$ holds for all $t$ in a subset $E_{r} \subseteq J$ of measure greater than $\delta / 3-2 / r^{2}$. Clearly $J \backslash \varliminf_{r \rightarrow \infty} E_{r}$ has measure zero. Therefore $T\left(t+h_{r}\right) x \rightarrow T(t) x$ almost everywhere in $J$. (This argument is due to Auerbach [2].) Let $t \in J$. There exists $t_{1}<t$ belonging to $J$ such that $T\left(t_{1}+h_{r}\right) x \rightarrow T\left(t_{1}\right) x$. Then

$$
T\left(t+h_{r}\right) x=T\left(t-t_{1}\right) T\left(t_{1}+h_{r}\right) x \rightarrow T\left(t-t_{1}\right) T\left(t_{1}\right) x=T(t) x
$$

by the assumed continuity of $T\left(t-t_{1}\right)$. Thus $T\left(t+h_{r}\right) x \rightarrow T(t) x$ everywhere in $J$. Since from any sequence $\left\{h_{k}\right\}$ tending to zero we may extract a subsequence $\left\{h_{k_{r}}\right\}$ with $\left|h_{k_{r}}\right|<\eta_{r}$, it follows that $T\left(t+h_{k}\right) x \rightarrow T(t) x$ everywhere in $J$. This completes the proof in the semigroup case.

The proof in the general case follows immediately by applying Theorem $\mathbf{1}^{\prime}$ to the semigroup $\{S(t)\}, t \in \mathbf{R}^{+}$, which is defined on the space $\mathbf{R} \times X$ by

$$
S(t)\left(\begin{array}{l}
s \\
x
\end{array}\right) \stackrel{\text { def }}{=}\left(\begin{array}{c}
s+t \\
U(t, s) x
\end{array}\right) .
$$

Corollary. Let $X$ be a subset of a Banach space. Suppose that for each $s \in \mathbf{R}, x \in X$, the map $t \mapsto U(t, s) x$ is weakly continuous from the right on $(0, \infty)$. Then the map $(t, s, x) \mapsto U(t, s) x$ is continuous on $(0, \infty) \times \mathbf{R} \times X$ with respect to the norm topology on $X$.

Proof. See [3, Theorem 5.2].

Remarks. 1. The proof of Theorem 1 bears some resemblance to that of Banach [4] of the result of Fréchet that every Lebesgue measurable real-valued solution $g$ of the functional equation

$$
g(s)+g(t)=g(s+t)
$$

is continuous, and thus of the form $g(t)=A t$ for some constant $A$. (This is a special case of Theorem 1 , since any $f$ satisfying (2) generates a semigroup on $\mathbf{R}$ given by $T(t) \tau=e^{g(t)} \tau$.)

2. In this case $X=\mathbf{R}$, Theorem 1 may also be proved by an argument used by Alexiewicz and Orlicz [1] in their proof of Fréchet's result. With the notation of the above proof, the function $f$ is measurable and thus approximately continuous almost everywhere in $(0, \infty)$; by the semigroup property and the continuity of $T(t)$ for $t \geqslant 0$ it follows that $f$ is approximately continuous everywhere in $(0, \infty)$, and hence $f$ is continuous (see Denjoy [11] and Looman [16]). It might be possible to extend this argument to arbitrary metric $X$.

3. There is an obvious modification of Theorem 1 to the case when the evolutionary process is defined only locally in $t$.

4. It is not in general possible to deduce that $(t, s, x) \mapsto U(t, s) x$ is continuous on $[0, \infty) \times \mathbf{R} \times X$, even if $t \mapsto U(t, s) x$ is continuous on $[0, \infty)$ for every $(s, x)$. (See Chernoff [5].)

All the other results in [3] may be generalised in a straightforward way to evolutionary processes using the transformation (1). We give as a sample two 
such generalisations and take the opportunity to weaken slightly the hypotheses of the corresponding results in [3, Theorems 5.1, 5.3] (the proofs are very similar).

THEOREM 2. Let $X$ be arbitrary. Suppose that for each $s \in \mathbf{R}, x \in X$, the map $t \mapsto U(t, s) x$ is Baire continuous on $(0, \infty)$ and, when restricted to the complement of some first category set, has second countable range. Then the map $(t, s, x)$ $\mapsto U(t, s) x$ is sequentially continuous on $(0, \infty) \times \mathbf{R} \times X$.

TheOREM 3. Let $X$ be a subset of a uniformly convex Banach space. Suppose that

(a) for each $s_{1}, s_{2} \in \mathbf{R}, x_{1}, x_{2} \in X, t_{n} \rightarrow 0+$ implies

$$
\liminf _{n \rightarrow \infty}\left\|U\left(t_{n}, s_{1}\right) x_{1}-U\left(t_{n}, s_{2}\right) x_{2}\right\| \leqslant\left\|x_{1}-x_{2}\right\|,
$$

(b) for each $s \in \mathbf{R}, x \in X$, the map $t \mapsto U(t, s) x$ is weakly continuous from the right at $t=0$.

Then for each $s \in \mathbf{R}, x \in X$, the map $t \mapsto U(t, s) x$ is continuous on $[0, \infty)$ with respect to the norm topology on $X$.

We remark that condition (i) in the definition of an evolutionary process is not needed for the validity of Theorems 1 and 2 . We remark also that there are useful methods of generating a semigroup from a given process other than by (1). (See Dafermos [9] and the references therein.) However, these methods, while having definite advantages over (1) for stability theory, do not improve our results.

4. Some counterexamples. Perhaps the simplest example of an evolutionary process is when $X=\mathbf{R}$ and each operator $U(t, s)$ is linear and defined on $\mathbf{R} \times \mathbf{R}$. Let $\{U(t, s)\}$ have the form

$$
U(t, s) r=e^{g(t, s)} r
$$

for some function $g: \mathbf{R} \times \mathbf{R} \rightarrow \mathbf{R} . g$ satisfies the functional equation

$$
g(t+\tau, s)=g(t, \tau+s)+g(\tau, s), \text { for all } t, \tau, s \in \mathbf{R} .
$$

The general solution of (4) is

$$
g(t, s)=h(t+s)-h(s),
$$

where $h: \mathbf{R} \rightarrow \mathbf{R}$ is arbitrary. It is therefore clear that, for example, neither strong measurability nor Baire continuity of $(t, s) \mapsto U(t, s) x, x \in X$, suffices to prove continuity of this map when (A) is replaced by an assumption of continuity of $U(t, s) x$ with respect to $x$ alone.

When $\{U(t, s)\}=\{T(t)\}$ is a semigroup then $g(t, s) \equiv g(t)$, where $f$ satisfies Cauchy's equation (2). In 1905 Hamel [14] showed using the axiom of choice that there are discontinuous solutions of (2). Thus even for semigroups of continuous linear operators on a Banach space Theorems 1 and 2 are false without the hypothesis of strong measurability or Baire continuity on the map $t \mapsto T(t) x$. Can this hypothesis be weakened to the requirement of precompactness of $T((\alpha, \beta)) x$ for all $\alpha, \beta \in \mathbf{R}^{+}$? This question is motivated by the 
result of Ostrowski [18], who, extending work of Darboux [10] and Sierpiński [21], showed that any solution $f$ of (2) which is bounded above on a set of positive measure is necessarily continuous (for an alternative proof see Kestelman [15]). The answer is no. For example, define $\{T(t)\}, t \in \mathbf{R}$, by

$$
\begin{gathered}
T(t)( \pm \pi / 2)= \pm \pi / 2, \\
T(t) \tau=\tan ^{-1}[g(t)+\tan \tau], \quad \tau \in(-\pi / 2, \pi / 2),
\end{gathered}
$$

where $g$ is any discontinuous solution of (2). It is easily checked that (6) defines a group of continuous (nonlinear) operators on $[-\pi / 2, \pi / 2]$ such that each nontrivial orbit is discontinuous (in fact, there will be just one orbit in $(-\pi / 2, \pi / 2)$ if and only if $g$ is bijective-such solutions $g$ to (2) are easy to construct using a Hamel basis of $\mathbf{R}$ over the rationals). $\{T(t)\}, t \in \mathbf{R}$, can be trivially extended to $\mathbf{R}$. Finally, we remark that $(S(t) \theta)(\tau)=\theta(T(t) \tau)$ for $\theta \in C([-\pi / 2, \pi / 2])$ defines a group $\{S(t)\}, t \in \mathbf{R}$, of linear isometries on $C([-\pi / 2, \pi / 2])$ with the maximum norm such that each nontrivial orbit is discontinuous.

ACKNOWLEDGEMENT. This paper was written while the author held part of a United Kingdom Science Research Council Research fellowship at the Lefschetz Center for Dynamical Systems, Brown University.

\section{REFERENCES}

1. A. Alexiewicz and W. Orlicz, Remarque sur l'équation fonctionelle $f(x+y)=f(x)+f(y)$, Fund. Math. 33(1945), 314-315. MR 8, 27.

2. H. Auerbach, Sur la relation $\lim _{h_{n} \rightarrow 0} f\left(x+h_{n}\right)=f(x)$, Fund. Math. 11(1928), 193-197.

3. J. M. Ball, Continuity properties of nonlinear semigroups, J. Functional Analysis 17 (1974), 91-103.

4. S. Banach, Sur l'équation fonctionelle $f(x+y)=f(x)+f(y)$, Fund. Math. 1(1920), $123-124$.

5. P. R. Chernoff, $A$ note on separate and joint continuity (to appear).

6. P. R. Chernoff and J. Marsden, On continuity and smoothness of group actions, Bull. Amer. Math. Soc. 76 (1970), 1044-1049. MR 42 \#19.

7. _- Infinite-dimensional Hamiltonian systems, Lecture Notes in Math., vol. 425, Springer-Verlag, Berlin, 1974.

8. M. G. Crandall and A. Pazy, Semi-groups of nonlinear contractions and dissipative sets, J. Functional Analysis 3(1969), 376-418. MR 39 \#405.

9. C. M. Dafermos, Semiflows associated with compact and uniform processes, Math. Systems Theory 8 (1974), 142-149.

10. M. G. Darboux, Sur le théorème fondamental de la géometrie projective, Math. Ann. 17(1880), 55-61.

11. A. Denjoy, Sur les fonctions dérivées sommables, Bull. Soc. Math. France 43(1915), 161-248.

12. N. Dunford, On one parameter group of linear transformations, Ann. of Math. (2) 39(1938), 569-573.

13. N. Dunford and J. T. Schwartz, Linear operators. I: General theory, Pure and Appl. Math., vol. 7, Interscience, New York, 1958. MR 22 \#302.

14. G. Hamel, Eine Basis alle Zahlen und die unstetigen Lösungen der Funktionalgleichung: $f(x+y)=f(x)+f(y)$, Math. Ann. 60(1905), 459-462.

15. H. Kestelman, On the functional equation $f(x+y)=f(x)+f(y)$, Fund. Math. 34 (1947), 144-147. MR 9, 188.

16. H. Looman, Sur deux catégories remarquables de fonctions de variable réelle, Fund. Math. 5(1924), 105-111.

17. J. von Neumann, Uber einen Satz von Herrn M. H. Stone, Ann. of Math. (2)33 (1932), 567-573. 
18. A. Ostrowski, Über die Funktionalgleichung der Exponential-funktion und verwandte Funktional-gleichungen, Jber. Deutsch. Math.-Verein 38 (1929), 54-62.

19. J. C. Oxtoby, Measure and category, Springer-Verlag, New York, 1971.

20. R. S. Phillips, On one-parameter semi-groups of linear transformations, Proc. Amer. Math. Soc. 2 (1951), 234-237. MR 12, 617.

21. W. Sierpiński, Sur une propriété des fonctions de M. Hamel, Fund. Math. 5 (1924), 334-335.

Lefschetz Center for Dynamical Systems, Division of Applied Mathematics, Brown University, Providence, Rhode Island 02912

Current address: Department of Mathematics, Heriot-Watt University, Edinburgh, Scotland 